\title{
Coincidence of Sarcoidosis With Ankylosing Spondylitis in a 48-Year-Old Woman; A Therapeutic Challenge
}

\author{
Gholamhossein Alishiri ${ }^{1}$, Ehsan Rahmanian ${ }^{2 *}$, Mahsa Ramezanpour ${ }^{2}$ \\ ${ }^{1}$ Chemical Injuries Research Center, Systems Biology and Poisonings Institute, Department of Rheumatology, Faculty \\ of Medicine, Baqiyatallah University of Medical Sciences, Tehran, Iran \\ ${ }^{2}$ Student Research Committee, Department of Rheumatology, Faculty of Medicine, Baqiyatallah University of Medical \\ Sciences, Tehran, Iran
}

*Corresponding Author: Ehsan Rahmanian, M.D., Fellowship of Rheumatology, Student Research Committee, Department of Rheumatology, Faculty of Medicine, Baqiyatallah University of Medical Sciences, Tehran, Iran. Tel: +98-9133533028, Email: drrahmanianehsan@yahoo.com

Received February 23, 2020; Accepted May 27, 2020; Online Published June 16, 2020

\begin{abstract}
Introduction: A case of pulmonary sarcoidosis is reported because of difficulties in diagnosis and treatment, including the co-existence of ankylosing spondylitis (AS) and severe corticosteroid dependence.

Case Presentation: A 48-year-old nonsmoking woman referred to the hospital because of chronic nonproductive cough and dyspnea with a 10-year history of AS. Bilateral rhonchi was detected in lung auscultation. There was a significant limitation in lumbar activity and range of motion in flexion (positive Schober's test), extension, and lateral bending. In lumbosacral magnetic resonance imaging (MRI), irregularities in the sacroiliac joint and bilateral sacroiliitis were evident. The angiotensin-converting enzyme level was elevated. Biopsy in hilar lymphadenopathy by transbronchial lung biopsy was done, and the histopathological findings showed chronic nonnecrotizing granulomatosis inflammation compatible with sarcoidosis. Anti-tumor necrosis factor drugs was effective on steroiddependent coexisting of sarcoidosis and AS. The symptoms were absent in regular follow up.

Conclusion: Pulmonary fibrosis due to sarcoidosis can be prevented by suitable treatment. Clinical trials are needed to confirm the impact of treatments with monoclonal antibodies against tumor necrosis factor (TNF), for curing sarcoidosis.

Keywords: Sarcoidosis, Ankylosing Spondylitis, Infliximab, Tumor Necrosis Factor, Pulmonary Fibrosis
\end{abstract}

\section{Introduction}

Sarcoidosis is a granulomatous multiple organinvolvement disease which has no definite etiology. Pathophysiology manifestation includes inflammatory reactions with aggregation of macrophages, which is the most specific finding in histology evaluations. Such chronic inflammatory particles and granulation can be seen in any vital organ, especially the heart and the lung. ${ }^{1}$

Managing pulmonary sarcoidosis is difficult because of four major concerns: $(i)$ it may be subclinical, without any damage to other organs or precise features; (ii) nearly $20 \%$ of cases may become aggravated and progress towards pulmonary fibrosis with an increased chance of death; (iii) there is no specific paraclinical index or test for determining the severity and prognosis of lung involvement; and (iv) treatment modalities, including steroids, methotrexate, and anti-tumor necrosis factor (TNF) drugs, have serious side effects. ${ }^{2-6}$ Considering these issues, clinical studies have reported multiple steps for curing pulmonary sarcoidosis and preventing pulmonary fibrosis. ${ }^{7}$ Researchers have shown that preventing low quality of life caused by pulmonary sarcoidosis is the main reason for clinical interventions. ${ }^{3}$

Corticosteroids are the drugs of first choice against the acute phase of pulmonary involvement; they can improve respiratory symptoms and radiological findings. Aside from their advantages, steroids beget many complications in therapeutic doses such as adrenal insufficiency, bone and mineral metabolism impairment, glucose intolerance, and predisposition to opportunistic infections.

For corticosteroid sparing treatment methods, methotrexate is proposed, while for last-ditch interventions, anti-TNF therapies, which have been evaluated by researchers, are used. ${ }^{8,9}$ An increased angiotensinconverting enzyme (ACE) level has no diagnostic value, and such elevation relates to the severity of the extra pulmonary disease and corticosteroid dependency. ${ }^{10}$

A literature search for two major keywords "sarcoidosis" and "ankylosing spondylitis" (AS) identified case reports for the most part. In PubMed (NLM), case reports from

Copyright (C) 2020 The Author(s). This is an open-access article distributed under the terms of the Creative Commons Attribution License (http:// creativecommons.org/licenses/by/4.0), which permits unrestricted use, distribution, and reproduction in any medium, provided the original work is properly cited. 
$1951^{11}$ to $2019^{12}$ were found. This shows that concurrent pulmonary sarcoidosis and AS could be valuable for presentation because of its scarcity, clinical and pathological features, and history as well as its management and treatment.

Kudrin et al reported on rheumatoid arthritis patients suffering sarcoid-like granulomatous disorder with respiratory and parotid involvement who were responsive to etanercept therapy. Etanercept is a TNF inhibitor, and Kudrin et al believed that anti-TNF therapy was the major cause of granulomatous lesion development. They emphasized that there is limited data on the antagonists of TNF in the case of sarcoidosis. ${ }^{13}$ O'Shea et $\mathrm{al}^{14}$ reported another case of pulmonary sarcoidosis after treatment with infliximab, an anti-TNF monoclonal antibody.

Despite the aforementioned evidence, the case of a woman suffering pulmonary sarcoidosis and AS who was responsive to infliximab is reported because of some difficulties in diagnosis and treatment regarding the correlation of the disease with AS, its superimposing to AS, and severe corticosteroid dependence.

\section{Case Presentation}

A 48-year-old nonsmoking woman referred to the pulmonary clinic of Baghiatollah hospital, Tehran, Iran, because of lasting nonproductive cough and dyspnea. She was a housewife with no exposure to infections and especially none to tuberculosis patients. She lived in an urban area, and her family members had no history of positive tuberculosis or purified protein derivative (PPD) test results. Her medical history revealed she had been evaluated for AS for 10 years and suffered chronic inflammatory back pain and morning stiffness as the beginning symptoms. She had been treated with indomethacin and sulfadiazine, but had poor compliance. In the physical examination of the patient, bilateral rhonchi was diagnosed during lung auscultation. Skin lesions were absent, and the other small joints were normal. Examination of both small and large joints revealed no positive point. The patient was significantly limited in lumbar activity, range of motion in flexion (positive Schober's test), extension, and lateral bending.

A chest X-ray (CXR) revealed an approximately normal parenchyma and hilar adenopathy. Irregularities in the sacroiliac joint and bilateral sacroiliitis were detected by lumbosacral magnetic resonance imaging (MRI). Spiral lung computed tomography (CT) was also done and revealed mild bilateral reticular lesions with a mosaic pattern. Bilateral hilar adenopathy was also detected (Figure 1).

In laboratory findings, mild anemia, normal liver and kidney function, and high erythrocyte sedimentation rate (ESR) and CRP were recorded. In complementary evaluations, elevated levels of the ACE was seen (Table 1). A whole body plethysmograph showed that the total lung capacity (TLC) was decreased, and restrictive changes were evident. TLC was decreased, while the fev1/fvc ratio (the ratio of forced expiratory volume in 1 second on forced vital capacity (FVC), also called the Tiffeneau-Pinelli Index) was approximately normal (Figure 2). ${ }^{15}$

The patient was a candidate for hilar lymphadenopathy transbronchial lung biopsy. The biopsy sample was sent to the surgical pathology lab and evaluated for histology/ cytology by tissue processing, hematoxylin and eosin staining, and immunohistochemistry staining for $\mathrm{CD}$ markers. The histopathological findings showed chronic non-necrotizing granulomatosis inflammation compatible with sarcoidosis (Figure 3; A and B: low magnification; C: high power field view). Acid-fast bacillus was not seen and cell determinant (CD) markers (CD30, CD15, CD20, CD3, CD45, and Ki-67) as well as epithelial membrane antigen (EMA) tests were all negative.

The most difficult concern was in scheduling the firstline treatment composed of a two-month administration of $30 \mathrm{mg} / \mathrm{d}$ of prednisolone. Gradually, the corticosteroid therapy was tapered, but the symptoms worsened. For the second step, the prednisolone dose was increased to $40 \mathrm{mg} / \mathrm{d}$, with tapering after two months, which was discontinued due to repeated-flair imaging. As a steroid-sparing therapy, the patient was treated with oral
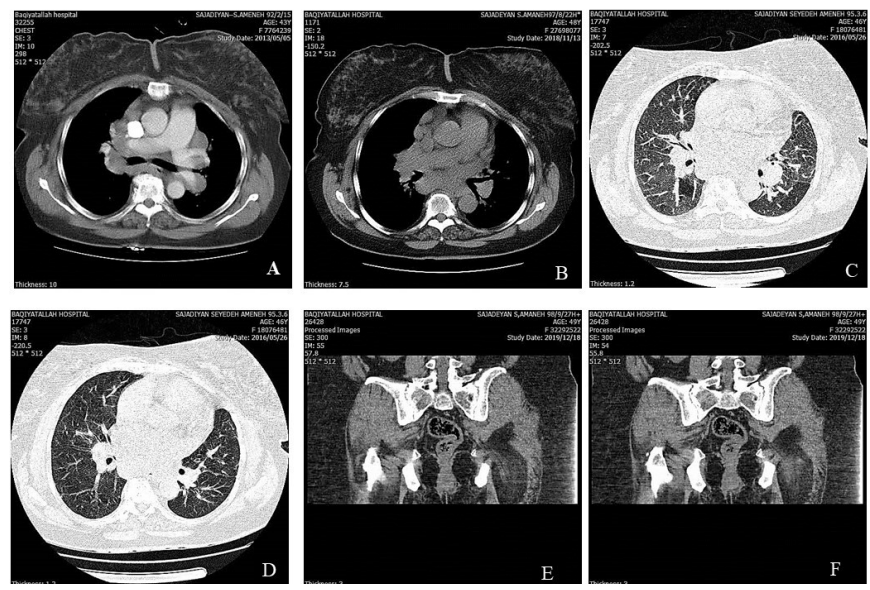

Figure 1. A-B: bilateral hilar adenopathy, mediastinal view; C-D: hilar adenopathy, and paranchimal involvement in base of the lung; E-F: pelvic CT scan, irregular sclerosis in SI joint assisted grade IV sacroiliitis 
Table 1. Laboratory findings of the Patients

\begin{tabular}{lcc}
\hline Index & Results & Normal Range \\
\hline $\mathrm{WBC}(\mathrm{cells} / \mu \mathrm{L})$ & 8500 & $4000-10000$ \\
$\mathrm{Hb}(\mathrm{gr} / \mathrm{dL})$ & 11.7 & $12-14$ \\
Platelet $\left({ }^{*} 1000\right)$ & 374 & $150-450$ \\
$\mathrm{BUN}(\mathrm{mg} / \mathrm{dL})$ & 9 & $7-20$ \\
Creatinine $(\mathrm{mg} / \mathrm{dL})$ & 0.7 & Less than 1.5 \\
$\mathrm{SGOT}(\mathrm{U} / \mathrm{L})$ & 13 & $10-40$ \\
$\mathrm{SGPT}(\mathrm{U} / \mathrm{L})$ & 16 & $7-50$ \\
$\mathrm{ESR}(\mathrm{mm} / \mathrm{h})$ & 42 & Less than 30 \\
$\mathrm{CRP}$ & 79 & Less than 40 \\
\hline
\end{tabular}

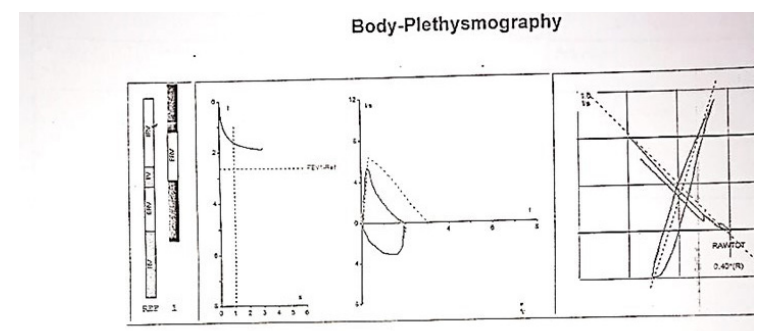

Spirometry, Flow-Volume

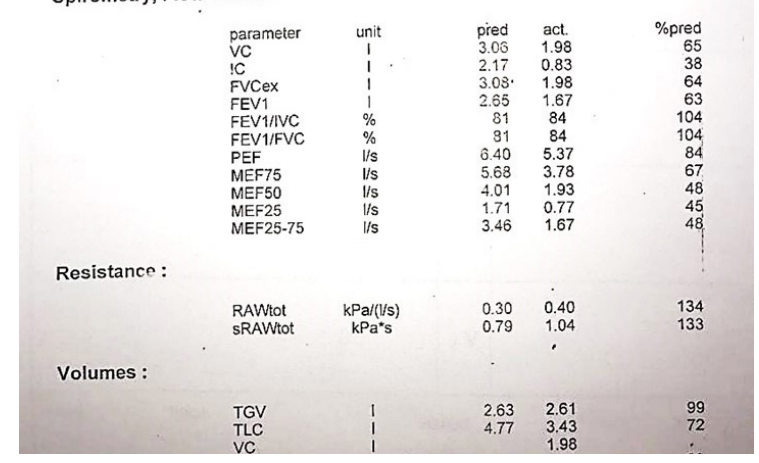

Figure 2. Pulmonary Function Test Assisted Mixed Pattern (Restrictive and Mild Obstructive) of Lung Involvement.

methotrexate $15 \mathrm{mg} / \mathrm{d}$, which was increased to $30 \mathrm{mg} / \mathrm{d}$; however, the symptoms relapsed when the steroid dosage was decreased. In the last treatment step, anti-TNF drugs were considered because of both sarcoidosis and AS. The PPD skin test was positive $(15 \mathrm{~mm})$, and the interferon gamma release assay was negative. Thus, isoniazid 300 $\mathrm{mg} / \mathrm{d}$ was administered after starting with infliximab. In regular follow up, the patient was symptom free.

\section{Discussion}

Pulmonary sarcoidosis needs treatment based on the degree of decreasing pulmonary function tests and disturbed quality of life. ${ }^{16}$ Indication of treatment was the severe and chronic cough plus dyspnea that impaired the quality of life of this presented case.

The treatment steps consisted of three major mainstays. First, $20-40 \mathrm{mg} / \mathrm{d}$ of prednisolone was prescribed for 1-3 months and then tapered to $5-10 \mathrm{mg} / \mathrm{d}$ as a maintenance dose for about 1 year for fatigue and microfibril neuropathy. As the second step of treatment, disease-modifying antirheumatic drugs, including methotrexate, azathioprine, leflunomide, hydroxychloroquine, and mycophenolate
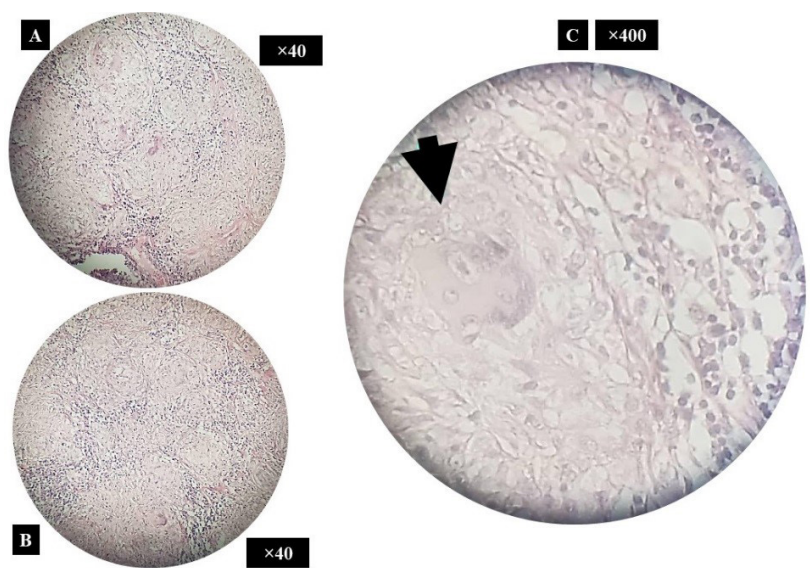

Figure 3. Microscopic Study of Lung Tissue Assisted With Chronic Nonnecrotizing Granulomatous Inflammation Compatible With Sarcoidosis

mofetil, were suggested. Finally, anti-TNF agents were recommended for refractory situations. Infliximab can improve dyspnea and forced vital capacity, while etanercept is not effective on pulmonary or ocular sarcoidosis. ${ }^{17}$

Raised ESR, pulmonary involvement (in CXR), and increased serum ACE activity were the major findings in the diagnostic orders of this patient. A definite diagnosis was established by hilar-lymphadenopathy biopsy. The findings confirmed similar studies about the role of biochemical markers in the diagnosis and prognosis of sarcoidosis. False positive results for ACE levels can be reported in primary biliary cirrhosis, alcoholic liver disease, and hyperthyroidism. ${ }^{18}$

One difficulty in management in the present case was the repeated flairs of symptoms when corticosteroid doses were tapered. This important issue led to the patient's therapeutic method being changed and the use of novel agents like infliximab. Because of the good efficacy of corticosteroids on pulmonary sarcoidosis, more antigranulomatous drugs have been suggested to decrease corticosteroid dependency and its side effects. Another benefit of these novel agents is that they can be used to treat patients who are resistant to regular steroid regimens. ${ }^{19}$

A dose higher than $10 \mathrm{mg}$ of prednisolone is not appropriate for daily treatment according to the majority of professional clinicians. Instead, they recommend the use of corticosteroid-sparing agents in pulmonary sarcoidosis, including methotrexate, azathioprine, adalimumab, and infliximab as the second or third remediation steps. ${ }^{20,21}$

The coexistence of AS and sarcoidosis has been reported in this case report with no obvious relationship. CDmarker positive lymphocytes have been declared as the common origin of both diseases, and patients with such coexistence can be more resistant to joint treatments, just like a case-report in India. ${ }^{22}$ A history of AS and new overriding sarcoidosis, which was presented in an Indian case as well as the current case, can change the treatment method and drive clinicians to use anti-TNF agents.

Wafa et al reported a case of this coexistence in Tunisia, and they also experienced problems with therapeutic 
methods. Ultimately, they recommended infliximab, as was done for the current patient. ${ }^{23}$ Based on previous evidence, attempts were made to manage the current patient with an anti-TNF agent and to follow her so as to evaluate her quality of life and respiratory symptoms. Valeyre et al presented a 34 year-old male patient with a history of cigarette smoking who had sarcoidosis with AS. They reported that infliximab was effective in treating the illness. The notable difference between our case and one presented by Valeyre et al was in the responsiveness to corticosteroids. Valeyre et al did not use corticosteroids for their patients because of normal results in respiratory function, whereas they were used in this case and found to be ineffective. ${ }^{24}$

Overall, a point for discussion is that patients suffering pulmonary sarcoidosis and concomitant AS may be treated safely with monoclonal antibody products, which could be useful for suppressing TNF-dependent inflammation and subsequent tissue reactions representing as sarcoidosis. The current report is evidence of successful remediation of an immunologic reaction with novel antibody-based medicine and opens a new window of hope to the next generation of therapies for sarcoidosis.

\section{Conclusion}

Pulmonary fibrosis due to sarcoidosis can be prevented by suitable treatment. Nevertheless, gathering the casereport data and establishing clinical trials are essential in confirming the efficiency of monoclonal antibody-based treatments for disorders such as sarcoidosis and related diseases. Furthermore, it should not be forgotten that each treatment process should be patient-centered. The authors believe that a fixed rule may not be essentially true for all patients with similar findings, as seen in the current case compared with previously published works.

\section{Authors' Contributions}

All Authors contributed equally to this Study.

\section{Conflict of Interest Disclosures}

The authors declare that they have no conflicts of interest.

\section{Ethical Approval}

The patient gave permission for the presentation of his case reports.

\section{Acknowledgments}

The authors would like to thank the "Clinical Research Development Unit" of Baqiyatallah Hospital for their kind cooperation.

\section{References}

1. James WE, Koutroumpakis E, Saha B, et al. Clinical features of extrapulmonary sarcoidosis without lung involvement. Chest. 2018;154(2):349-356. doi:10.1016/j.chest.2018.02.003.

2. Judson MA. The clinical features of sarcoidosis: a comprehensive review. Clin Rev Allergy Immunol. 2015;49(1):63-78. doi:10.1007/ s12016-014-8450-y.
3. Judson MA. Quality of life in sarcoidosis. Semin Respir Crit Care Med. 2017;38(4):546-558. doi:10.1055/s-0037-1602589.

4. Chopra A, Kalkanis A, Judson MA. Biomarkers in sarcoidosis. Expert Rev Clin Immunol. 2016;12(11):1191-1208. doi:10.1080/1 744666x.2016.1196135.

5. Kwda A, Gldc P, Baui B, et al. Effect of long term inhaled corticosteroid therapy on adrenal suppression, growth and bone health in children with asthma. BMC Pediatrics. 2019;19(1):411. doi:10.1186/s12887-019-1760-8.

6. Judson MA. Developing better drugs for pulmonary sarcoidosis: determining indications for treatment and endpoints to assess therapy based on patient and clinician concerns. F1000Res. 2019;8. doi:10.12688/f1000research.20696.1.

7. Moller DR. Negative clinical trials in sarcoidosis: failed therapies or flawed study design? Eur Respir J. 2014;44(5):1123-1126. doi:10.1183/09031936.00156314.

8. Panselinas E, Judson MA. Acute pulmonary exacerbations of sarcoidosis. Chest. 2012;142(4):827-836. doi:10.1378/ chest.12-1060.

9. Vorselaars AD, Crommelin HA, Deneer VH, et al. Effectiveness of infliximab in refractory FDG PET-positive sarcoidosis. Eur Respir J. 2015;46(1):175-185. doi:10.1183/09031936.00227014.

10. Belhomme N, Jouneau S, Bouzillé $G$, et al. Role of serum immunoglobulins for predicting sarcoidosis outcome: a cohort study. PLoS One. 2018;13(4):e0193122. doi:10.1371/journal. pone.0193122.

11. Verstraeten JM, Bekaert J. [Association of ankylosing spondylitis and sarcoidosis]. Acta Tuberc Belg. 1951;42(2):149-151.

12. Nnodum BN, Hariri LP, Mavrommati D, Dudley L. A case of severe symptomatic central nervous system sarcoidosis secondary to treatment with adalimumab. Case Rep Rheumatol. 2019;2019:7121539. doi:10.1155/2019/7121539.

13. Kudrin A, Chilvers ER, Ginawi A, et al. Sarcoid-like granulomatous disease following etanercept treatment for RA. J Rheumatol. 2007;34(3):648-649.

14. O'Shea FD, Marras TK, Inman RD. Pulmonary sarcoidosis developing during infliximab therapy. Arthritis Rheum. 2006;55(6):978-981. doi:10.1002/art.22351

15. Minelli R. Appunti dalle lezioni di fisiologia umana. La Goliardica Pavese; 1992

16. Judson MA. Strategies for identifying pulmonary sarcoidosis patients at risk for severe or chronic disease. Expert Rev Respir Med. 2017;11(2):111-118. doi:10.1080/17476348.2017.1281745.

17. James WE, Judson MA. Therapeutic strategies for pulmonary sarcoidosis. Expert Review of Respiratory Medicine. 2020;14(4):391-403. doi:10.1080/17476348.2020.1721284.

18. Kahkouee S, Samadi K, Alai A, Abedini A, Rezaiian L. Serum ACE level in sarcoidosis patients with typical and atypical HRCT manifestation. Pol J Radiol. 2016;81:458-461. doi:10.12659/ pjr.897708.

19. Judson MA, Baughman RP, Costabel U, Mack M, Barnathan ES The potential additional benefit of infliximab in patients with chronic pulmonary sarcoidosis already receiving corticosteroids: a retrospective analysis from a randomized clinical trial. Respir Med. 2014;108(1):189-194. doi:10.1016/j.rmed.2013.11.019.

20. Schutt AC, Bullington WM, Judson MA. Pharmacotherapy for pulmonary sarcoidosis: a Delphi consensus study. Respir Med. 2010;104(5):717-723. doi:10.1016/j.rmed.2009.12.009.

21. Vorselaars ADM, Wuyts WA, Vorselaars VMM, et al. Methotrexate vs azathioprine in second-line therapy of sarcoidosis. Chest. 2013;144(3):805-812. doi:10.1378/chest.12-1728.

22. Rahul CD, Sharma RK, Talwar D. Sarcoidosis with ankylosing spondylitis: changing therapeutic landscape. Sarcoidosis Vasc Diffuse Lung Dis. 2018;35(3):285-288. doi:10.36141/svdld. v35i3.6679.

23. Wafa H, Saoussen M, Dhia K, Imen Z, Montacer KM. Sarcoidosis and spondyloarthritis: a coincidence or common etiopathogenesis? Caspian J Intern Med. 2018;9(1):100-103. doi:10.22088/ cjim.9.1.100.

24. Valeyre D, Soler P, Clerici C, et al. Smoking and pulmonary sarcoidosis: effect of cigarette smoking on prevalence, clinical manifestations, alveolitis, and evolution of the disease. Thorax. 1988;43(7):516-524. doi:10.1136/thx.43.7.516 